\title{
COMPARATIVE ASSESSMENT OF MAXILLOFACIAL MORPHOLOGY IN PATIENTS WITH BILATERAL CLEFT LIP AND PALATE USING CONE BEAM COMPUTED TOMOGRAPHY
}

\author{
Yomna Elhoseiny", Raghdaa Mostafa**, Marwa ElKassaby*** and Mona Abou El-Fotouh***
}

\begin{abstract}
Objective: The purpose of this study was to investigate maxillofacial morphology using CBCT with the ultimate goal of finding whether differences existed in a two suggested subtypes of nonsyndromic bilateral cleft lip and palate (BCLP) patients.

Design: This retrospective study included CBCT data of 22 BCLP patients with mean age of 9.6 years. The patients were divided initially according to premaxillary characteristics into two groups and this classification was further confirmed by measurement of sella-nasion- point A angle (SNA), Group (P): This represent BCLP characterized by well-developed (P) prominent pre-maxilla and SNA $>80 \pm 2$, Group(R): This represent BCLP characterized by ill-developed (R) rudimentary pre-maxilla and $\mathrm{SNA}<80 \pm 2$. The relation between maxilla and mandible measured by point A-nasion-B point angle (ANB), angle of septal deviation (ASD) and anterior upper facial height (AUFH) were assessed using On-demand 3D software and was compared among the two groups.
\end{abstract}

Results: Patients within group P showed significantly higher ANB ( $\mathrm{P}$ value $\leq 0.001$ ). Patients within group R showed significantly higher ASD angle ( $\mathrm{P}$ value $\leq 0.05$ ), while AUFH showed insignificant difference between the two groups ( $P$ value $\geq 0.05)$.

Conclusions: Maxillofacial morphology measurements assessed in this study support that difference exist between the two investigated subtypes of non-syndromic BCLP.

KEYWORDS: Bilateral cleft lip and palate, Premaxilla, premaxillary protrusion, CBCT

\footnotetext{
* Teaching Assistant in Oral and Maxillofacial Radiology, Faculty of Dentistry, Ain Shams University, Cairo, Egypt ** Lecturer of Oral and Maxillofacial Radiology, Faculty of Dentistry, Ain Shams University, Cairo, Egypt

*** Professor of Oral and Maxillofacial Surgery, Head of Oral and Maxillofacial Surgery Department, Faculty of Dentistry, Ain Shams University Cairo, Egypt

**** Professor of Oral and Maxillofacial Radiology, Head Oral Medicine, Periodontology, and Oral Radiology

Department, Faculty of Dentistry, Ain Shams University, Cairo, Egypt
} 


\section{INTRODUCTION}

Cleft lip and palate (CLP) represent the most common oromaxillofacial congenital anomalies that affect one in 500 to one in 1000 newborns worldwide. It occurs around the $36^{\text {th }}$ or $37^{\text {th }}$ day of gestation due to failure of fusion between different embryonic processes ${ }^{(1-4)}$. The exact etiology of CLP is unknown but it has been shown that it occurs as combination of environmental and genetic factors during the early stages of pregnancy ${ }^{(5)}$.

The presence of an orofacial cleft causes physical and functional disturbances to the craniofacial complex that affect individuals both physically and psychologically ${ }^{(6,7)}$. Multiple anatomical variations are seen in CLP patients as midfacial hypoplasia, hypoplastic maxillary sinus, deviated nasal septum and various dental anomalies ${ }^{(8-10)}$.

Complete BCLP is a severe cleft subtype that represents a substantial challenge both clinically and surgically. However, it is important to note that not all anatomical irregularities and dysmorphology associated with BCLP can be detected clinically such as deviated nasal septum, reduced nasal chamber width and asymmetry of maxilla and mandible. Since these defects can impair feeding, mastication, speech, and dentofacial development, it is important to detect them radiographically ${ }^{(11)}$.

CBCT images allow visualization and measurement of anatomical landmarks, linear distances, angles, surface areas, and airway/sinus volumes at any site within the craniofacial complex ${ }^{(12-16)}$. Also it provides $2 \mathrm{D}$ and $3 \mathrm{D}$ view of the defect in cleft patients.

The general term "BCLP" is usually a misleading description as not all bilateral cases shows the same premaxillary characteristics and general features. Different presentations of the defect affect the response of cases to presurgical orthopedics and primary surgical repair of lip ${ }^{(11)}$.
To our knowledge, one attempt in dental literature could be found to differentiate between various presentations of non- syndromic BCLP ${ }^{(11)}$.

In this study, a clinically oriented descriptive classification for BCLP was suggested dividing BCLP patients into two subtypes; Group P characterized by well-developed $(\mathrm{P})$ prominent premaxilla and Group R characterized by ill-developed (R) rudimentary pre-maxilla However, this study included only infants before and after primary lip repair.

Therefore, the current study was conducted in an attempt to investigate whether differences in the maxillofacial morphology exist between the two suggested subtypes of BCLP patients using CBCT.

\section{METHODS}

It was a retrospective study of CBCT data of patients recruited from the data base of the Cleft Care Center affiliated to the Oral and Maxillofacial Surgery Department at Ain shams University, Cairo, Egypt. Our study was exempted from the Review Ethics Committee as all included CBCT scans were made anonymous and were taken for reasons other than the purpose of this study.

The inclusion criteria for the cases included CBCT data of non-syndromic BCLP patients who had undergone previous CLP surgeries as follows: The lip was repaired by Millard- type lip repair at about 3months of age, the palate was repaired by push back palatoplasty and the Furlow method between 9-18 months of age. The exclusion criteria were one or both of the following: (1) a history of surgical or orthodontic interference for repositioning of the premaxilla. (2) CBCT scans that shows any artifacts that interfered with the identification of the required landmarks. Selection of the patients scans were based on previously recorded chart information. Patient's gender and age at the time of the scan were also recorded from the charts. A total of 22 scans matched the inclusion criteria and were not excluded by the exclusion criteria. 
The CBCT images were all taken with the same iCAT 3D dental imaging system (i-CAT; Imaging Sciences International, Hatfield, PA) at $120 \mathrm{kVp}$ and $5 \mathrm{~mA}$, with a scanning time of 4 to 5 seconds and a voxel size of $0.377 \mathrm{~mm}$, using an amorphous silicon flat panel sensor and a $16-\mathrm{cm}$ field of view. Data were stored in the Digital Imaging and Communications in Medicine (DICOM) file format.

Using On-demand 3D application software, scans were reoriented to standardized orthogonal views. They were classified initially according to premaxillary characteristics assessed visually in the axial, coronal, sagittal views and 3D volume into two groups; Group P characterized by welldeveloped prominent $(\mathrm{P})$ pre-maxilla and Group $\mathrm{R}$ characterized by ill-developed rudimentary $(\mathrm{R})$ premaxilla.

This Classification was further confirmed by measuring the SNA angle which demonstrates the relation between the maxilla and the cranial base, and indicates whether the maxilla has normal, prognathic, or retrognathic position ${ }^{(17)}$.

To measure SNA angle, certain anatomical landmarks were identified; point $\mathrm{S}$, nasion, and point A (Table 1). Then the angle was measured using angle tool on sagittal slice which was adjusted from coronal view as follows: sequential slices were

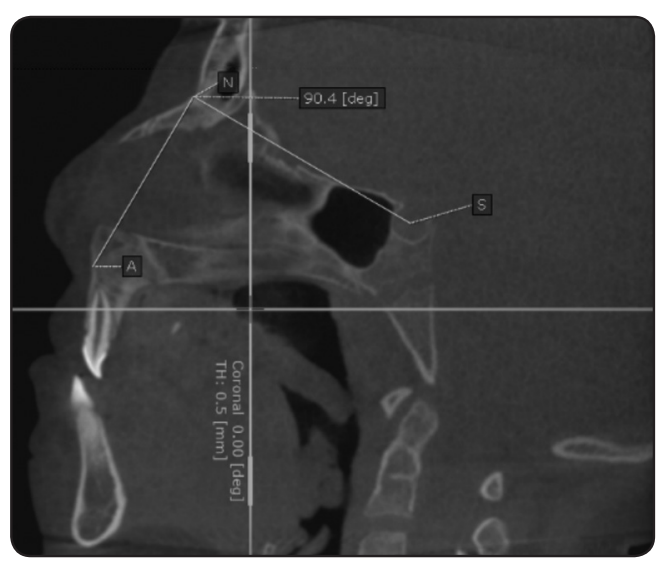

Fig. (1) Sagittal image showing SNA angle $\mathrm{S}=$ sella; $\mathrm{N}=$ nasion; $\mathrm{A}=$ point $\mathrm{A}$ examined till the reference sagittal plane is passing through middle of crista galli posteriorly and between lower central incisors anteriorly (Figure 1). Group P included scans with SNA $>80 \pm 2$ while, group R included scan with SNA $<80 \pm 2$.

Three maxillofacial morphological variables were assessed in this study. First, the relation between the maxilla and the mandible which was assessed through measuring ANB angle; (A point, nasion, B point) (Table 1). This angle was measured on the same sagittal slice used in measuring the SNA angle. The required anatomical landmarks were identified then the required angle was measured using angle tool (Figure 2). ANB indicates whether the skeletal relationship between the maxilla and the mandible is a normal skeletal class I, a skeletal Class II, or a skeletal class III relationship ${ }^{(18)}$.

Second, Septal deviation was assessed by measuring angle of septal deviation (ASD), which is defined as the angle between midline and line passing from the top point of the nasal septum to the most convex point of the deviated nasal septum (point O) ${ }^{(19)}$. Coronal slices were sequentially viewed and the slice showing the most deviated part of the nasal septum was selected, on which both the top point of the nasal septum and point $\mathrm{O}$ were identified, then a line was drawn joining the

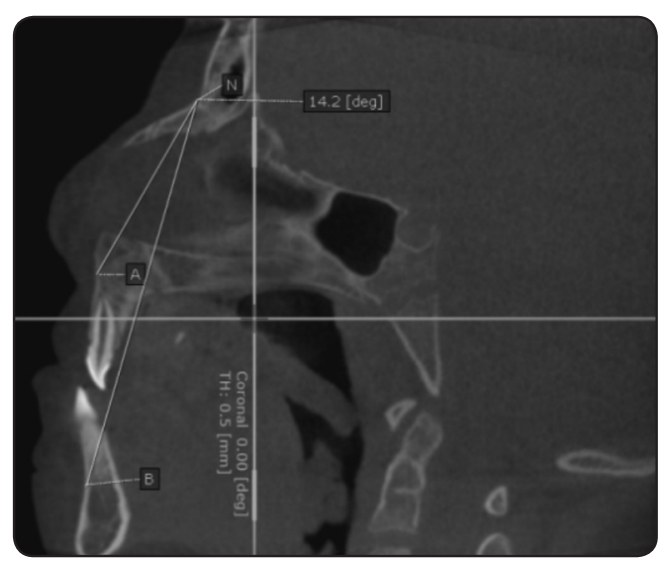

Fig. (2) Sagittal image showing ANB angle, $A=$ point $A ; N=$ nasion; $\mathrm{B}=$ point $\mathrm{B}$, 
2 points. Angle between midline and this line was measured using angle tool (Figure 3).

Third, anterior upper facial height (AUFH) was measured in millimeters. It was defined as the distance between nasion and anterior nasal spine (N-ANS) ${ }^{(20)}$. It was measured on the same sagittal slice used for measuring SNA angle. The required landmarks were identified; nasion and ANS (Table 1) then, the distance of the straight line joining the two points was calculated (Figure 4).

Identification of anatomical landmarks is demanding in young CLP patients at the mixed dentation stage. Therefore, all measurements were repeated two times with 1 month interval by the same investigator and their average values were used in the further analysis.

\section{Statistical analysis}

The statistical analysis was performed using GraphPad prism 7.05 for Windows, GraphPad Software, La Jolla California USA and Microsoft Excel. The data were expressed as mean and standard deviation (SD). Unpaired-student $t$ test was used to compare the three maxillofacial morphometric variables among the two groups. Intra-observer agreement was assessed using Alpha (Cronbach) reliability analysis. The statistically significant level was set at $\mathrm{P} \leq .05$.

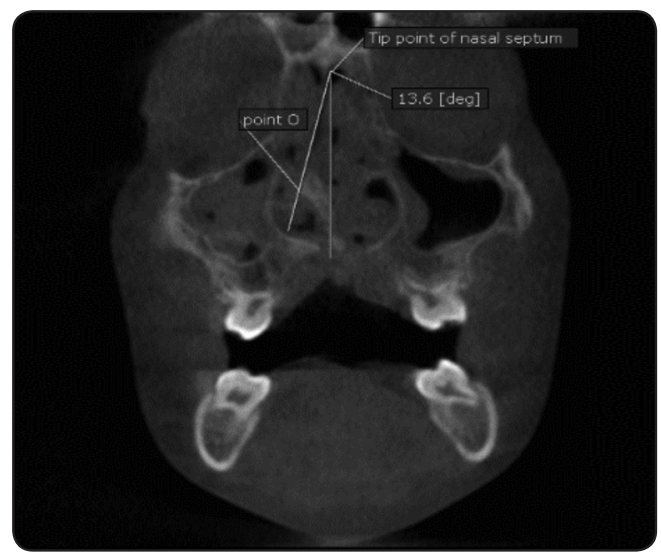

Fig. (3) Coronal image showing angle of septal deviation, $\mathrm{O}=$ most convex point of the deviated nasal septum, ASD $=13.6^{\circ}$
TABLE (1) Anatomical land marks

\begin{tabular}{|c|l|}
\hline \multicolumn{2}{|l|}{ Anatomical Land mark definitions } \\
\hline 1 & $\begin{array}{l}\text { A point (sub spinal): most concave point of anterior } \\
\text { maxilla. }\end{array}$ \\
\hline 2 & Nasion: most anterior point on frontonasal suture. \\
\hline 3 & $\begin{array}{l}\text { B point (supramentale): most concave point on } \\
\text { mandibular symphysis. }\end{array}$ \\
\hline 4 & Sella: mid-point of sella turcica. \\
\hline 5 & $\begin{array}{l}\text { Point O: most convex point of the deviated nasal } \\
\text { septum }\end{array}$ \\
\hline
\end{tabular}

\section{RESULTS}

A total of 22 patients scan (12 scans in group $\mathrm{P}$ and 10 scans in group $\mathrm{R}$ ) were included in this study from both genders, with a mean age of 9.6 years. Table 2 shows the means, standard deviations and 2 tailed $\mathrm{T}$ test results of the three measurements. ANB angle showed a higher mean value in group $\mathrm{P}$ $(12.4 \pm 4.58)$ compared to group $\mathrm{R}(3.1 \pm 4.67)$, the difference was significant $(\mathrm{P}$ value $\leq 0.001)$.

A significant difference was also found in the angle of septal deviation (ASD) among the two groups ( $\mathrm{P}$ value $\leq 0.05)$. Group $\mathrm{P}$ showed lower degree of septal deviation compared to group

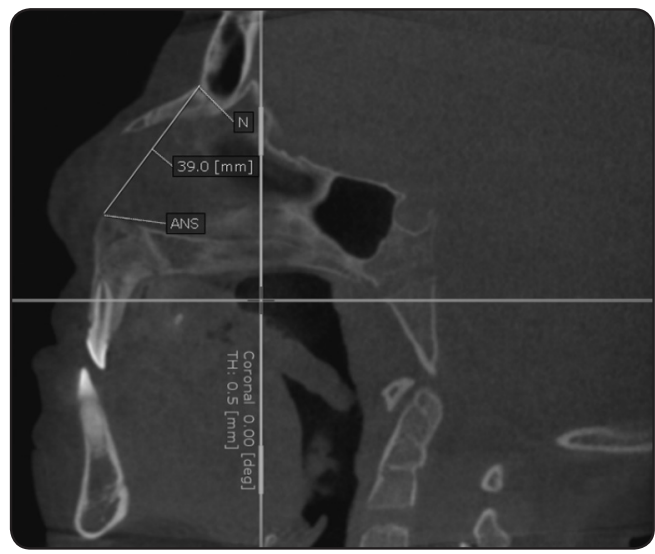

Fig. (4) Sagittal image showing AUFH measured as straight line from $\mathrm{N}=$ nasion to ANS = anterior nasal spine using ruler tool, $\mathrm{AUFH}=39 \mathrm{~mm}$ 
R. Concerning AUFH, group $\mathrm{P}$ showed higher mean value compared to group $\mathrm{R}(47.44 \pm 6.02$, $43.94 \pm 4.71)$ respectively, yet the difference was not statistically significance $(\mathrm{P}$ value $\geq 0.05)$. Cronbach's alpha ranged from good to excellent for all measurements, this indicates that the method of assessment produced consistent and reproducible results (Table 3)

TABLE (2) Means, standard deviations and 2 tailed $\mathrm{T}$ test results for ANB angle, ASD angle and AUFH

\begin{tabular}{|c|c|c|c|c|}
\hline Variable & Groups & Mean & SD & P value \\
\hline \multirow{2}{*}{ ANB } & $\mathrm{P}$ & 12.4 & 4.58 & \multirow{2}{*}{$0.0006^{* * *}$} \\
\cline { 2 - 4 } & $\mathrm{R}$ & 3.1 & 4.67 & \\
\hline \multirow{2}{*}{ ASD } & $\mathrm{P}$ & 9.66 & 4.05 & \multirow{2}{*}{$0.0495^{*}$} \\
\cline { 2 - 4 } & $\mathrm{R}$ & 13.3 & 4.01 & \\
\hline \multirow{2}{*}{ AUFH } & $\mathrm{P}$ & 47.44 & 6.02 & \multirow{2}{*}{0.3402} \\
\cline { 2 - 4 } & $\mathrm{R}$ & 43.94 & 4.71 & \\
\hline
\end{tabular}

*Indicates statistical significant: $P \leq 0.05$

TABLE (3) Alpha (Cronbach) reliability analysis showing intra-observer reliability

\begin{tabular}{|l|l|}
\hline Variable & Cronbach's Alpha \\
\hline ASD & 0.874 (Good) \\
\hline ANB & 0.985 (Excellent) \\
\hline AUFH & 0.954 (Excellent) \\
\hline
\end{tabular}

\section{DISCUSSION}

Premaxillary characteristics are not similar in all BCLP cases due to the difference in premaxillary position since birth, where it is fixed to the vomer bone apically leading to abnormalities in the position. The premaxilla may be protruding and prominent or rudimentary and hypoplastic ${ }^{(11 ; 21)}$. Its posi- tion is also influenced by pressure from the tongue and lip together with forward growth of the midline structures and the lateral processes ${ }^{(22 ; 23)}$.

A study conducted by Elkassaby MA, et al., 2013, tested the response of BCLP neonate patients to presurgical orthopedics and primary lip repair based on premaxillary characteristics. A significant morphological difference was found between the two subtypes of BCLP, however the authors concluded that more studies are required to validate the differences during different growth periods ${ }^{(11)}$.

Therefore the objective of the current study was to compare the maxillofacial morphology among the two suggested subtypes of non- syndromic BCLP patients in mixed dentation stage to detect if difference excited using CBCT.

Patients with history of surgical or orthodontic interference for repositioning of the premaxilla were excluded as in case of early manipulation of protrusive premaxilla (at the time of lip closure) the anteroposterior growth of the premaxilla and consequently SNA angle is unfavorably affected due to drastic effects on maxillary growth ${ }^{(24)}$.

Identification of anatomical landmarks is challenging in young CLP patients owing to abnormal anatomy and presence of tooth germs molding the anterior contour of the maxilla. This especially holds true for the localization of the landmarks: ANS and point $\mathrm{A}^{(25)}$. Therefore, all measurements were repeated two times with 1 month interval by the same investigator.

In the present study, the ANB angle was measured to compare the relation between the maxilla and the mandible among the two subtypes, a statistically significant difference $(\mathrm{P}>0.01)$ was found. Group $\mathrm{P}$ showed higher mean values indicating sagittal Angle class I or II division crossbite while, group $\mathrm{R}$ showed sagittal Angle class III jaw relationship. This result may be due to different positions of the premaxilla in the two groups. 
A previous study by Gnoinski WM, and Rutz G, 2009 assessed multiple cephalometric measurements on 29 BCLP subjects (23 males, 6 females), at the ages of 5, 10, and 15 years. All patients were subjected to the same treatment protocol set by the University of Zurich. ANB angle was measured to assess sagittal intermaxillary relationship, the group average value at the age of 5 was (13.1), yet three subjects showed values clearly below the group average values. On comparing ANB angle of those subjects, at age 15 , to average group values they showed negative angles indicating tendency for sagittal Angle class III jaw relationship, while the remaining subjects showed tendency for sagittal Angle class I or II division ${ }^{(26)}$.

Moreover, a study by Bittermann GK, et al., 2015 conducted a systematic review from the period 1960 to 2015 to analyze different presentation and management of the premaxilla in BCLP and reported that in BCLP the premaxilla shows extreme abnormalities in the position and most often is protruding (27).

In the present study, the angle of septal deviation (ASD) was measured as CLP patients presented uneven nasal septum morphologic patterns therefore, it is of great clinical value in the longterm multidisciplinary treatment of the patients ${ }^{(19)}$.

Two methods were reported for measuring ASD. One method was suggested by Jiang M, et al., 2014, in their study, ASD was measured as the angle between midline and line passing from the top point of the nasal septum to the most convex point of the deviated nasal septum (point $\mathrm{O}$ ) ${ }^{(19)}$. The other method, measured septal deviation at three different levels; at the cartilaginous septum (anterior nasal spine), bony septum (posterior nasal spine), and midpoint between the anterior and posterior nasal spine and then identified the point of maximal septal deviation for all patients ${ }^{(28)}$. Yet we choose the first method to evaluate the deviation for better reproducibility and convenience.
Several previous studies investigated the degree and direction of septal deviation in various cleft palate and/or alveolus forms and compared it to normal population ${ }^{(19 ; 28-30)}$ but to the best of our knowledge; none of these previous studies utilized ASD to compare between the two subtypes of BCLP.

In the current study, ASD showed a statistically significant difference between group $\mathrm{P}$ and group $\mathrm{R}$, where septal deviation in group $\mathrm{R}$ was more severe. This could be attributed to the theory that the nasal septum has a significant role in downward and forward displacement of the maxilla and that the size of premaxilla in BCLP is partially affected by the nasal septum ${ }^{(31 ; 32)}$.

Our result was in accordance with Bansal A, et al., 2012, in their study of nasal septal deviation in total of 80 non cleft subjects with various relation of maxilla to cranial base (normal relation, retruded maxilla, protruded maxilla), the septal deviation was found to be more common among subjects with retruded maxilla than subjects with protruded maxilla and as a result the authors assumed that in patients with retruded maxilla, nasal septum could be the reason for deficient maxillary growth in sagittal plane ${ }^{(33)}$.

Regarding AUFH, a non-significant difference between the two groups was found; however group $\mathrm{P}$ showed higher mean values compared to group $\mathrm{R}$. This finding suggest that growth retardation in group $\mathrm{R}$ is more severe compared to group $\mathrm{P}$.

Growth deficiency is demonstrated in all CLP patients and is more severe in bilateral medial facial dysplasia ${ }^{(34)}$. It may be a consequence to surgical intervention or an inherent aspect of CLP ${ }^{(35)}$.

In our study, the two subtypes of BCLP showed variations in the maxillofacial morphology. These variations may affect results of treatment and necessitate modifications in treatment planning. Different handling protocols may be needed for the patients with different subtypes of BCLP, 
where Surgical maxillary advancement via orthodontic functional devices, orthognathic surgery or distraction osteogenesis are required in case of rudimentary hypoplastic premaxilla ${ }^{(36)}$, while in case of protruding premaxilla, presurgical orthopedics, orthodontics or surgical repositioning of the premaxilla might be required ${ }^{(11)}$.

Future studies should include longitudinal data to assess over time if the morphological differences would remain and whether using different handling protocols based on this classification would affect the outcome.

\section{CONCLUSION}

The ANB was significantly higher while the ASD was significantly lower in group $\mathrm{P}$ than group $\mathrm{R}$. Moreover, AUFH showed no significant difference among the two groups. Within the limitation of this study, we can conclude that the two suggested subtypes of non-syndromic BCLP showed different maxillofacial morphology

\section{Funding}

The author(s) received no financial support for the research, authorship, and/or publication of this article.

\section{Declaration of Conflicting Interests}

The author(s) declared no potential conflicts of interest with respect to the research, authorship, and/or publication of this article

\section{REFERENCES}

1- Weinberg SM, Naidoo SD, Bardi KM, Brandon CA, Neiswanger K, Resick JM, Martin RA, Marazita ML. Face shape of unaffected parents with cleft affected offspring: Combining three dimensional surface imaging and geometric morphometrics. Orthod Craniofac Res 2009; 12:271-281.

2- Lee JK, Park JW, Kim YH, Baek SH. Association between PAX9 single-nucleotide polymorphisms and nonsyndromic cleft lip with or without cleft palate. J Craniofac Surg 2012; 23:1262-1266.
3- Marazita ML. The evolution of human genetic studies of cleft lip and cleft palate. Annu Rev Genomics Hum Genet 2012; 13:263-283.

4- Choi YK, Park SB, Kim YI, Son WS. Three-dimensional evaluation of midfacial asymmetry in patients with nonsyndromic unilateral cleft lip and palate by cone-beam computed tomography. Korean J Orthod 2013; 43:113-119.

5- Setó-Salvia N, Stanier P. Genetics of cleft lip and/or cleft palate: association with other common anomalies. Eur J Med Genet 2014 Aug; 57(8):381-93.

6- Hozyasz K. The search for risk factors that contribute to the etiology of non-syndromic cleft lip with or without cleft palate $(\mathrm{CL} / \mathrm{P})$ in the Polish population.Pediatr Pol 2010; 85 (6): 609-623

7- Pereira AV, Nuno Fradinho, Sara Carmo, Juliana M. de Sousa, David Rasteiro, Regina Duarte and Maria J. Associated Malformations in Children with Orofacial Clefts in Portugal: A 31-Year Study. Plast Reconstr Surg Glob Open. 2018 Feb; 6(2): e1635.

8- Freeman AK, Mercer NS, Roberts LM. Nasal asymmetry in unilateral cleft lip and palate. J Plast Reconstr Aesthet Surg 2013; 66: 506-512.

9- Hasanzadeh N, Majidi MR, Kianifar H, Eslami N. Facial softtissue morphology of adolescent patients with nonsyndromic bilateral cleft lip and palate. J Craniofac Surg 2014; 25:314-317.

10- Mangione F, Nguyen L,Foumou N, Bocquet E, Dursun E. Cleft palate with/without cleft lip in French children: radiographic evaluation of prevalence, location and coexistence of dental anomalies inside and outside cleft region. Clin Oral Investig. 2018 Mar; 22(2):689-695.

11- Elkassaby MA, Abdelrahman NI, Abbass IT. Premaxillary characteristics in complete bilateral cleft lip and palate: a predictor for treatment outcome. Ann Maxillofac Surg 2013; 3:11-19.

12- Aboudara C, Nielsen I, Huang JC, Maki K, Miller AJ, Hatcher D. Comparison of airway space with conventional lateral headfilms and 3-dimensional reconstruction from cone-beam computed tomography. Am J Orthod Dentofacial Orthop2009; 135:468-479.

13- Lenza MG, Lenza MM, Dalstra M, Melsen B, Cattaneo PM. An analysis of different approaches to the assessment of upper airway morphology: A CBCT study. Orthod Craniofac Res2010; 13:96-105. 
14- Ghoneima A, Kula K. Accuracy and reliability of conebeam computed tomography for airway volume analysis. Eur J Orthod 2013; 35:256-261.

15- AL-Fahdawy M. Cone beam computed tomography analysis of the pharyngeal airway in individuals with non-syndromic cleft:retrospective study]doctor thesis[.]Cairo [: Ain Shams University; 2015.

16- El-Badawy F. Comparison between Two computer simulation systems for volumetric analysis of alveolar bone grafts using Cone beam computed tomography] doctor thesis [.] Cairo [: Ain Shams University; 2015

17- Atit M, Deshmukh SV, RahalkarJ, Subramanian V, Naik C, Darda M. Mean values of Steiner, Tweed, Ricketts and McNamara analysis in Maratha ethnic population: A cephalometric study. APOS Trends in Orthodontic .2013 September 3(5): 137-151.

18- Arruda MBP. Angle Class III malocclusion with anteroposterior and vertical discrepancy in the final stage of growth. Dental Press J Orthod. 2017 May-Jun; 22(3): 109-118.

19- Jiang M, You M, Wang S, Wang K, Feng B, Wang H. Analysis of Nasal Septal Deviation in Cleft Palate and/or Alveolus Patients Using Cone-Beam Computed Tomography. Otolaryngol Head Neck Surg. 2014 Aug; 151(2):226-31

20- Folaranmi N and Isiekwe M. Anterior Face Height Values in a Nigerian Population. Ann Med Health Sci Res. 2013 Oct-Dec; 3(4): 583-587.

21-Ahmed O. Effect of lip muscles on maxillary growth in cleft lip and palate patients: Electromyographic study. master thesis[.]cairo[:Ain Shams University;2014.

22- Brouns J, Egyedi P. Osteotomy of the premaxilla. J Maxillofac Surg 1980; 8:182-186

23- Latham RA. Development and structure of the premaxillary deformity in bilateral cleft lip and palate. Br J Plast Surg 1973; 26:1-11.

24- Bishara SE, Olin WH. Surgical repositioning of the premaxilla in complete bilateral cleft lip and palate. Angle Orthod. 1972; 42:139-147.

25- Bongaarts CA, van ‘t Hof MA, Prahl-Andersen B, KuijpersJagtman AM. Identification of cephalometric landmarks in unilateral cleft lip and palate patients: are there alternatives for point A, ANS, and PNS?. Cleft Palate Craniofac J. 2008 Jan; 45(1):81-6.
26- Gnoinski WM, Rutz G. A longitudinal cephalometric study from age 5 to 18 years on individuals with complete bilateral cleft lip and palate. J Craniofac Surg. 2009 Sep; 20 Suppl 2:1672-82.

27- Bittermann GK, de Ruiter AP, Janssen NG, Bittermann AJ, van der Molen AM, van Es RJ, Rosenberg AJ, Koole R. Management of the premaxilla in the treatment of bilateral cleft of lip and palate: what can the literature tell us?. Clin Oral Investig. 2015 sep 16.

28- Massie JP, Runyan CM, Stern MJ, Alperovich M, Rickert SM, Shetye PR, Staffenberg DA, Flores RL. Nasal Septal Anatomy in Skeletally Mature Patients with Cleft Lip and Palate. JAMA Facial Plast Surg. 2016 Sep 1; 18(5):347-53.

29- Bayerlein T, Proff P, Koppe T, Fanghaenel J, Hosten N. Cartilaginous septum deviation in children with cleft lip, alveolus and palate--an MRI analysis. J Craniomaxillofac Surg. 2006 Sep; 34 Suppl 2:49-51.

30-Starbuck JM, Friel MT, Ghoneima A, Flores RL, Tholpady S, Kula K. Nasal airway and septal variation in unilateral and bilateral cleft lip and palate. clin Anat 2014 ;27(7):9991008.

31- Friede H, Morgan P. Growth of the vomero-premaxillary suture in children with bilateral cleft lip and palate.A histological and roentgencephalometric study. Scand J Plast Reconstr Surg. 1976; 10:45-55.

32- Vargervik K. Growth characteristics in the premaxilla and orthodontic treatment principles in bilateral cleft lip and palate. Cleft Palate J. 1983; 20:289-302.

33- Bansal A, Utreja AK, Satinder SP, Panda NK, Jena AK. The Association between Nasal Septum Deviation and Sagittal Maxillary Growth Discrepancy. Jp-journals. 2012 April; 2:10028-1021.

34- Noordhoff MS, Huang CS, Lo LJ. Median facial dysplasia in unilateral and bilateral cleft lip and palate: A subgroup of median cerebrofacial malformations. Plast Reconstr Surg 1993; 91:996-1007.

35- Naqvi ZA, BM Shivalinga BM, Ravi S, Munawwar S. Effect of cleft lip palate repair on craniofacial growth. J Orthod Sci. 2015 Jul-Sep; 4(3): 59-64.

36- Alyamani A, Abuzinada S.A protocol used to manage maxillary hypoplasia in cleft lip and palate patients. Open Journal of Stomatology 2012; 2, 130-135. 